\title{
Objectifs et mise en œuvre de l'instrumentation du projet d'extension en Mer de Monaco
}

\section{Camille KAPELA ${ }^{1,2}$, Michael LECOEUR ${ }^{3}$, Olivier GAY ${ }^{4}$}

1. BUGO3E -Segment Ports, 15 avenue du Centre, CS 20538 Guyancourt, 78286 SaintQuentin-en-Yvelines Cedex, France.

camille.kapela@egis.fr

2. ARTELIA, 6 rue de Lorraine, 38130 Echirolles, France.

camille.kapela@artelia.fr

3. BUGO3E -Segment Géotechnique, 33-43 avenue Georges Pompidou, BP 13115, 31131 Balma Cedex, France.

mickael.lecoeur@egis.fr

4. BUGO3E -Segment Géotechnique, 3 rue du Docteur Schweitzer, 38180 Seyssins, France.

olivier.gay@egis.fr

\section{Résumé :}

La Principauté de Monaco est l'état le plus densément peuplé au monde. Pour contrer l'exiguiité de son territoire, depuis le $\mathrm{XX}^{\mathrm{ème}}$ siècle, la Principauté a mis en œuvre des projets d'extension en mer tels que celui de l'Anse du Portier. Ce projet, initié en 2015, consiste en la construction d'un remblai d'une surface de 6 hectares, sur une hauteur maximum de 50 mètres, maintenu en place derrière une ceinture de 18 caissons en béton préfabriqués.

Pour contrôler l'amplitude des tassements de la plateforme et des caissons de l'Infrastructure Maritime, un programme d'instrumentation spécifique au projet, réparti en quatre étapes clés, a été mis en place. Chacune des quatre étapes identifiées comporte un suivi adapté. Il est en effet nécessaire de suivre les tassements primaires, secondaires et l'état de consolidation des sédiments au niveau du remblai principal dans le but de confirmer les analyses de liquéfaction réalisées lors de la phase de conception du projet, mais aussi d'améliorer la maîtrise du calcul des tassements qui doivent répondre à des critères très stricts. Il faut également pouvoir surveiller les tassements des remblais rapportés ainsi que les déplacements des caissons et des blocs de quai.

Cet article détaille les objectifs et le programme d'instrumentation du projet d'extension en Mer de Monaco par le biais d'une présentation des suivis pour chacune des quatre étapes évoquées et d'une analyse de la première phase d'instrumentation.

Mots-clés :

Instrumentation, Travaux maritimes, Remblaiement en mer, Tassements, Consolidation. 


\section{Thème 3 - Instrumentation, mesures, imagerie et télédétection}

\section{Introduction}

D'ici 2025, le premier éco-quartier de Monaco verra le jour, situé sur un remblai maritime. Afin d'assurer la pérennité de l'ouvrage et suivre son évolution au cours des différentes phases de travaux, la mise en place d'un programme d'instrumentation spécifique au projet devient essentielle. Cet article présente l'instrumentation mise en œuvre pour répondre aux objectifs de suivi des remblais et des caissons du projet d'extension en Mer de Monaco. Les hypothèses ayant permis d'établir le programme d'instrumentation sont présentées ainsi que les premiers retours liés à l'implémentation de la première phase.

\section{Présentation du projet}

\subsection{Général}

Le projet d'extension en mer de Monaco consiste en la réalisation d'une plateforme de six hectares derrière une ceinture de dix-huit caissons trapézoïdaux en béton préfabriqués (figures 1 et 2). Après une phase de dragage jusqu'au substratum, dans le but d'assurer une plateforme stable permettant de préserver la stabilité des caissons après leur installation, différentes phases de remblaiement ont lieu. L'ensemble des aménagements et des superstructures reposera sur des fondations profondes ancrées au substratum.

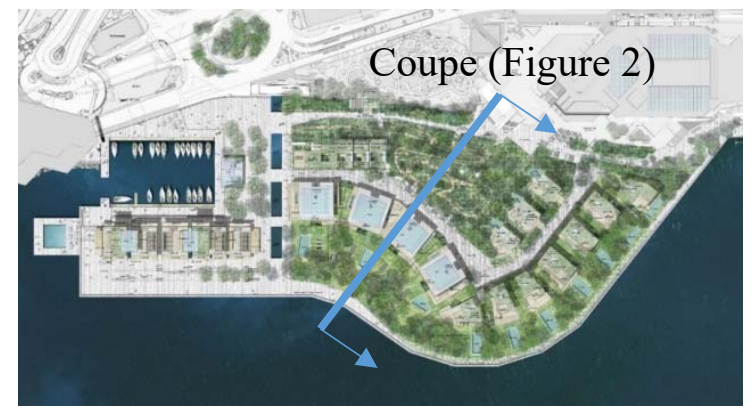

Figure 1. Vue du projet (VALODE \& PISTRE, 2014)

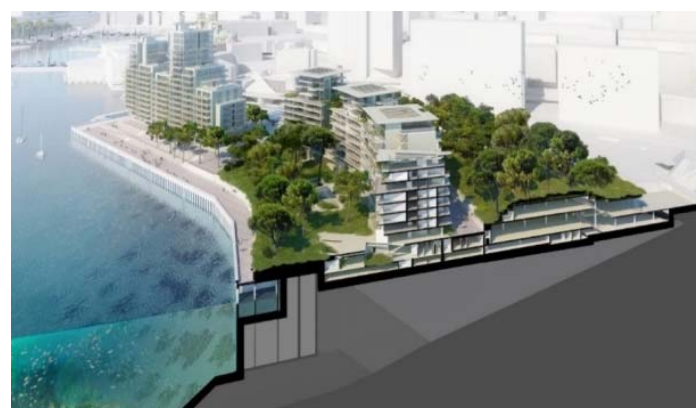

Figure 2. Coupe (VALODE \& PISTRE, 2014)

Les remblais sont de deux types :

a) Remblai en matériaux granulaires de carrière de granulométrie $20 / 180 \mathrm{~mm}$ pour l'assise des caissons, qualifié de "remblai technique" ;

b) Remblai en matériaux granulaire de carrière de granulométrie $0 / 20$ et $0 / 50 \mathrm{~mm}$ pour le remblaiement de la plateforme et qualifié de "remblai courant".

\subsection{Contexte géologique et géotechnique}

Les fonds marins sont constitués de sédiments quaternaires meubles recouvrant un substratum rocheux irrégulier, calcaire ou marno-calcaire suivant les zones, avec 


\section{XVIèmes Journées Nationales Génie Côtier - Génie Civil \\ Le Havre 2020}

localement la présence de brèche / éboulis / rognons. Les formations sédimentaires apparaissent soit limono-sableuses/limono-argileuses soit sableuses.

Le projet est soumis à un aléa sismique moyen et est traversé par une faille (la faille du Larvotto), justifiant également la mise en place d'un programme d'instrumentation

\subsection{Phasage des travaux}

Illustrées par le biais d'une coupe type du projet (figure 3), les principales phases de construction du projet sont présentées ci-après.

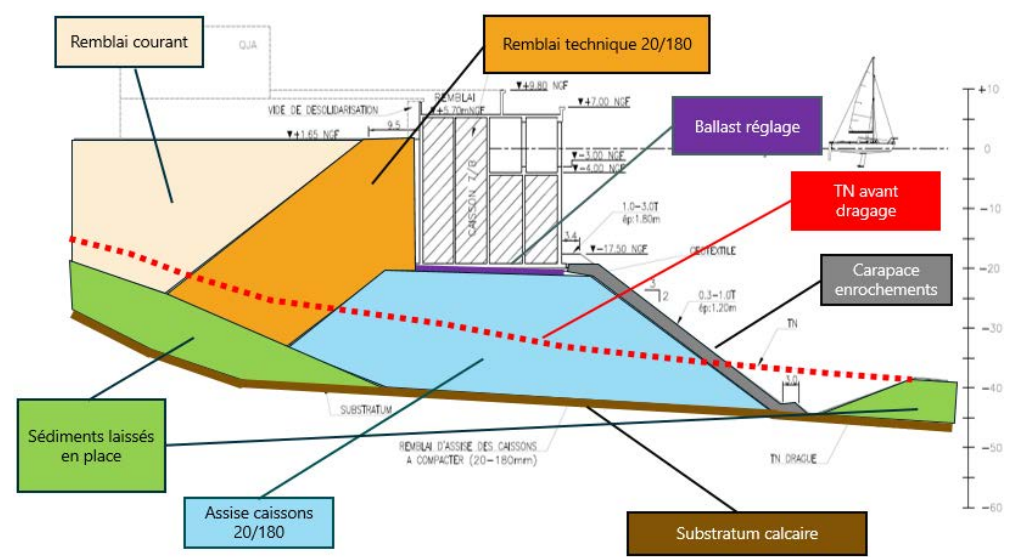

Figure 3. Coupe type du projet (Bouygues TP).

a) Purge des sédiments sous les caissons.

b) Mise en place du remblai d'assise des caissons (de même nature que le remblai technique) et de la première phase du remblai technique. Ces remblais sont vibrocompactés.

c) Mise en place des caissons.

d) Installation de la deuxième phase du remblai technique et vibrocompactage.

e) Mise en place du remblai courant et vibrocompactage.

f) Mise en place de colonnes ballastées, drains sismiques et jet grouting vis-à-vis du risque de liquéfaction des sols.

g) Réalisation des pieux pour la réalisation des fondations profondes.

h) Suivi d'exploitation de la plateforme.

\section{Enjeux de l'instrumentation}

Afin de répondre aux objectifs de suivi des remblais et des déformations des caissons, les enjeux suivants ont été pris en compte pour l'établissement du programme:

a) Objectifs en matière de tassements des sols en place (figure 4). Le site du projet est constitué de plusieurs zones selon des critères de tassements résiduels à 50 ans à atteindre définis par le client.

b) Phasage des travaux du projet ; 


\section{Thème 3 - Instrumentation, mesures, imagerie et télédétection}

c) Contexte géologique et géotechnique ;

d) Etat des différents capteurs disponibles et précisions des mesures ;

e) Durabilité des ouvrages (minimum 100 ans).

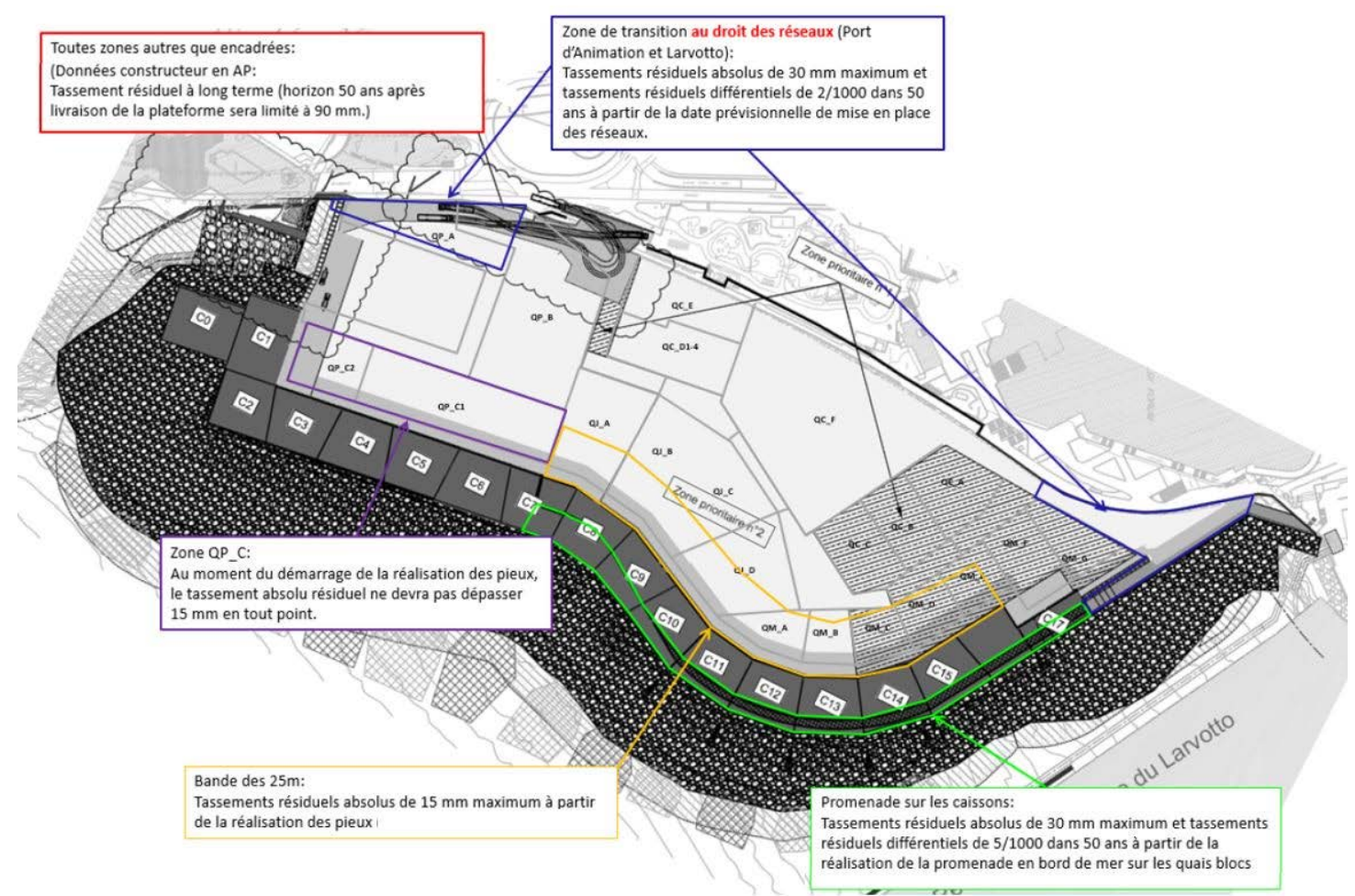

Figure 4. Critères de réception en tassements totaux (Bouygues TP).

Sur cette base, le programme d'instrumentation permet d'effectuer différents suivis pendant les travaux mais aussi pendant la phase d'exploitation du projet:

a) Suivi des tassements des sédiments au niveau de la plate-forme,

b) Suivi des tassements des remblais courants et techniques,

c) Suivi des déplacements des caissons,

d) Suivi des tassements et de la consolidation des sédiments, afin de pouvoir confirmer les analyses de liquéfaction et maîtriser le calcul de prédiction des tassements attendus.

\section{Contraintes liées aux travaux}

Certaines contraintes spécifiques liées aux travaux concernant le projet d'extension en mer de Monaco doivent impérativement être considérées à la fois pour la mise en place du programme d'instrumentation (conception du dispositif général d'instrumentation et pose des capteurs/équipement) et lors de l'interprétation des données collectées :

a) Travail nearshore et offshore, les profondeurs des fonds marins pouvant atteindre plus de $50 \mathrm{~m}$ à quelques mètres du rivage ;

b) Réalisation des pieux nécessaires pour les superstructures ; 


\section{XVIèmes Journées Nationales Génie Côtier - Génie Civil \\ Le Havre 2020}

c) Traitements des sols par colonnes ballastées, drains sismiques et jet grouting ;

d) Vibrocompactage des remblais ;

e) Co-activité importante sur site ;

f) Circulation des engins;

g) Pose de la dalle.

\section{Etapes du programme d'instrumentation}

Etant donné les contraintes liées au site et aux travaux, il est nécessaire de définir un plan pour chaque phase d'instrumentation.

Quatre étapes clés ont été identifiées et ont nécessité un suivi adapté :

a) Phase I : Instrumentation après dragage et avant remblaiement avec l'installation de 42 tassomètres. Les tassomètres ne sont pas pris en compte en termes de mesures pour les phases suivantes car il est envisagé qu'ils soient inexploitables suite aux travaux de vibrocompactage.

b) Phase II : Instrumentation après remblaiement et avant compaction. Lors de cette phase, sont mis en place :

o 14 extensomètres multipoints ancrés au substratum (longueur maximum de $50 \mathrm{~m}$ )

o 6 capteurs de pressions interstitielles

o 31 cibles topographiques pour plateforme

060 repères topographiques pour caissons

04 piézomètres

o 5 inclinomètres d'une longueur maximum de $25 \mathrm{~m}$.

Dans le cas où ces équipements seraient toujours exploitables après cette étape, il sera utilisé lors des étapes ultérieures. Dans le cas contraire, le processus d'instrumentation prévoit de les remplacer à l'identique (type d'acquisition et profondeur).

c) Phase III : Instrumentation après réalisation des pieux et avant la dalle avec l'installation de onze extensomètres multipoints en plus du dispositif d'instrumentation précédemment cité.

d) Phase IV : Instrumentation après réalisation des pieux et pendant l'exploitation avec le même programme d'instrumentation que la phase 3 hors cibles topographiques pour plateforme. Les extensomètres et capteurs de pression interstitielle sont équipés de câbles afin d'éviter une intervention humaine pendant l'exploitation. Les mesures par inclinomètres et repères topographiques pour caissons sont réalisées par une équipe spécialisée.

Le système d'acquisition de données combine des technologies filaires ou sans fil et des mesures spécifiques manuelles telle que la topographie. 


\section{Thème 3 - Instrumentation, mesures, imagerie et télédétection}

\section{Analyse de l'instrumentation de la phase I de la zone QPC}

\subsection{Plan d'instrumentation}

La zone QPC est la zone du projet pour laquelle le critère de tassement est le plus strict. Après démarrage des pieux, le tassement résiduel à 50 ans ne peut excéder $15 \mathrm{~mm}$. 18 des 42 tassomètres (43\%) sont installés dans la zone QPC depuis le mois de janvier 2018.

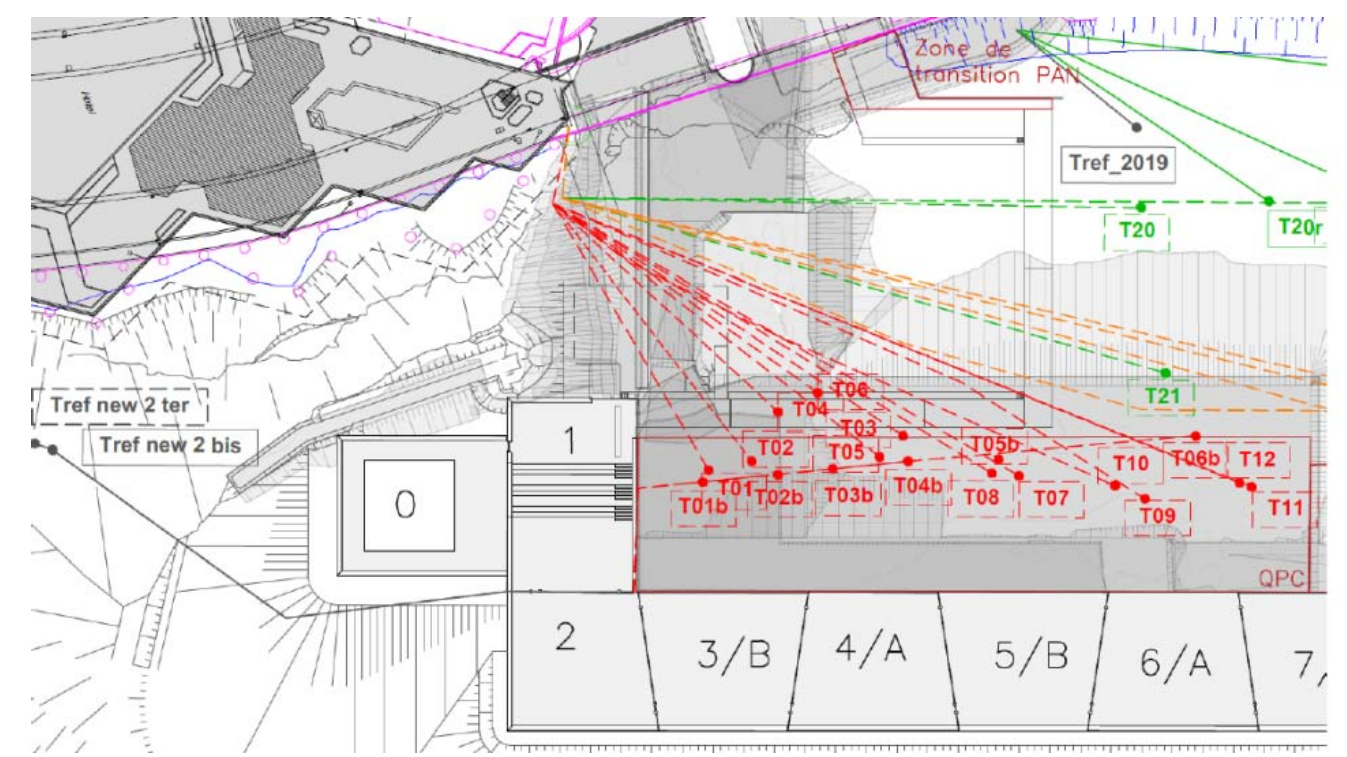

Figure 5. Plan d'implantations des 18 tassomètres sur QPC.

Des tassomètres ont été installés avant la première phase de mise en place du remblai technique (en 2018) et avant la seconde phase de remblaiement (en 2019). Ces tassomètres sont répartis de manière homogène dans la zone QPC et permettent donc d'avoir une estimation de l'état de consolidation atteint par les couches sous-jacentes de sédiments. Les tassomètres de référence (Tref), situés en dehors de la zone du chantier, permettent d'effectuer les corrections liées à la houle et à la marée. Ils représentent le niveau d'eau enregistré en fonction du temps.

Le dispositif d'acquisition consiste en une mesure toutes les 30 minutes via une centrale d'acquisition, reliée aux tassomètres par câbles.

\subsection{Méthode d'interprétation et modèle de consolidation}

La mesure de pression est effectuée en milieu maritime. Ainsi, la variation de pression au niveau des capteurs de pression n'est pas seulement induite par le tassement du massif, mais aussi par la marée et la houle ainsi que d'autres phénomènes à échelle globale ou locale dont la pression barométrique contribue de façon majoritaire. Afin de s'affranchir de ces effets, les mesures des capteurs sont moyennées sur 12 heures (soit 24 mesures) et les tassomètres de référence sont fixés au substratum, ne subissant pas de tassement. Pour 


\section{XVIèmes Journées Nationales Génie Côtier - Génie Civil \\ Le Havre 2020}

déduire le tassement, la hauteur d'eau mesurée au droit du capteur à traiter est retranchée de la hauteur d'eau mesurée au droit d'un capteur de référence.

Ensuite, un modèle de consolidation (sous charge constante) est ajusté aux résultats. Pour cela, le tassement est calculé comme la somme d'un tassement instantané et d'un tassement de consolidation. Le tassement instantané correspond au tassement des couches de matériaux sableux. Le tassement de consolidation est lié au tassement des couches plus argileuses. Le tassement instantané ainsi que la date à laquelle ce tassement est atteint sont estimés graphiquement pour chaque tassomètre étudié. Au-delà de la date à laquelle le tassement instantané est atteint, et sous charge constante, une courbe d'évolution type "consolidation" apparait. Le tassement de consolidation est écrit comme le produit de la variable U (définie dans la théorie de la consolidation, LEROUEIL et al., 1985) par la valeur de tassement finale reliée à de la consolidation.

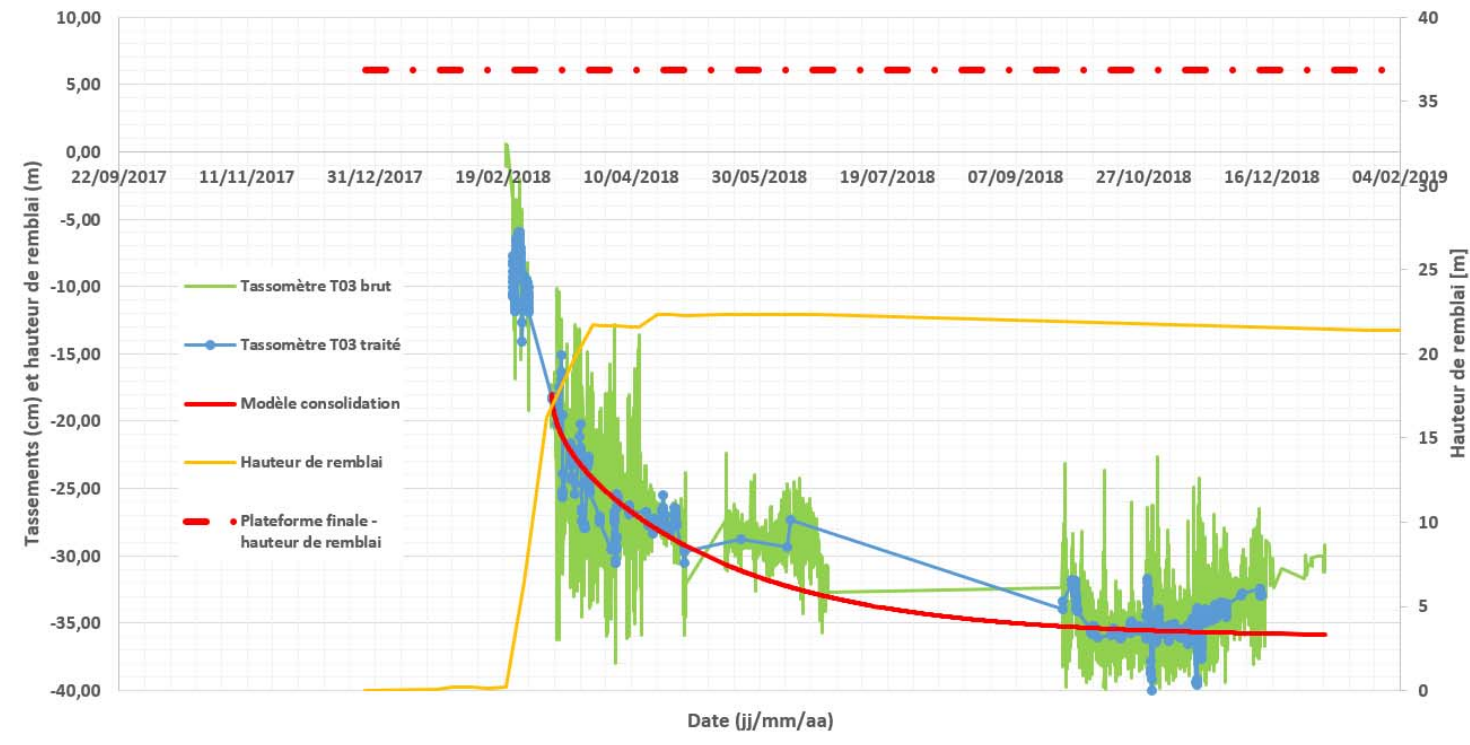

Figure 6. Tassements bruts/traités et modèle de consolidation pour un tassomètre.

6.3 Challenges rencontrés et recommandations

Certaines contraintes ont été observées :

a) Tassomètres de référence hors service à plusieurs reprises en raison des travaux sur les émissaires ayant lieu en dehors de l'emprise du terre-plein. Le site étant encadré par deux réserves naturelles, il était impossible de décaler les capteurs de référence.

b) En raison de mesures trop espacées, la houle n'a pas pu être discrétisée, sa fréquence étant de 4 à 7 secondes.

c) Des écarts par rapport aux tassements calculés ont parfois été observés. Ils peuvent être expliqués par les incertitudes liées aux mesures (précision, influence de la houle et de la marée, perte du capteur de référence, etc.) et par les incertitudes sur l'épaisseur et la nature des sédiments au droit des tassomètres. 


\section{Thème 3 - Instrumentation, mesures, imagerie et télédétection}

d) Rupture de certains câbles lors de la mise en œuvre des remblais, mettant hors service certains tassomètres.

Malgré les points cités précédemment, des analyses complémentaires ont permis d'exploiter la mesure et ainsi de la confronter aux approches théoriques. Quelques recommandations peuvent ainsi être partagées :

a) La houle et la marée en Méditerranée sont deux phénomènes pouvant représenter des variations de niveau d'eau de plusieurs dizaines de centimètres. La mesure des tassements étant réalisée via des transducteurs de pression qui mesurent une colonne d'eau et cherchant à caractériser des tassements centimétriques à pluri-centimétriques, il est très important de s'affranchir des effets de la houle et de la marée pendant la mesure de tassement.

b) La complexité de la réalisation et de l'exploitation des mesures tassométriques réside dans le maintien opérationnel des capteurs en phases travaux (et notamment remblaiement sous eau à une certaine profondeur) et surtout le maintien des capteurs de références permettant de s'affranchir de l'effet de la marée et de certains effets parasites. Il est conseillé d'avoir un capteur de référence secondaire permettant de garantir l'acquisition permanente des mesures.

c) Afin de mesurer les effets de la houle, l'acquisition des données serait plus adaptée avec une centrale dont les caractéristiques permettraient d'échantillonner en dessous de la période de la houle.

\section{Conclusions}

Le projet d'extension en mer de Monaco a été doté d'un plan d'instrumentation conséquent et inédit pour un projet de remblaiement maritime. A ce jour, seules les deux premières phases ont été mises en œuvre par le biais de l'installation de tassomètres et d'extensomètres. L'analyse de la zone QPC a montré qu'en dépit des challenges rencontrés, les tassements se stabilisent dans le temps. Cette tendance a été confirmée par les premières données des extensomètres de la seconde phase d'instrumentation.

Il est de toute importance, notamment sur les projets à activités multiples de mener une réflexion sur la fiabilisation en amont de tout le process instrumentation, de la position des capteurs de référence pour les pérenniser, comment doubler les références, fiabiliser les chemins de câbles ainsi que les contrôles et changements de capteurs pour ne pas avoir de pertes de données sur des phases clés de remblaiement.

\section{Référence bibliographique}

LEROUEIL S., MAGNAN J.-P., TAVENAS F. (1985). Remblais sur argiles molles. LCPC, Lavoisier, Paris, 360 p. 